\title{
Десятилетний опыт пневматической баллонной дилатации пищеводно-желудочного перехода
}

\section{А. В. Малиновский, М. Н. Майоренко, Н. Н. Чернов, М. Ю. Шигимага, Д. В. Кудинов \\ Одесский национальный медицинский университет \\ Ten-year experience of pneumatic balloon dilatation of esophago-gastric junction}

\author{
A. V. Malynovskyi, M. N. Majorenko, N. N. Chernov, M. J. Shigimaga, D. V. Kudinov
}

Odessa National Medical University

\section{Реферат}

Цель. Выбор оптимального режима пневматической баллонной дилатации (ПБД) пищеводно-желудочного перехода под рентгенологическим контролем при ахалазии (АП) и стенозе (СП) пищевода после пластики пищеводного отверстия диафрагмы (ПОД) и фундопликации.

Материалы и методы. С 2007 по 2017 г. ПБД выполнена 34 пациентам: 7 пациентам с I-II стадией АП; 13 - с рецидивом после лапароскопической кардиомиотомии (ЛКМТ); 14 - с функциональной дисфагией или СП после пластики ПОД и фундопликации.

Результаты. Осложнений ПБД не было. У всех пациентов достигнута ремиссия на протяжении разных сроков, зависевших от причины дисфагии. Возникшие рецидивы проанализированы и соотнесены с результатами повторных операций. Выводы. ПБД высокоэффективна и не сопровождается осложнениями при тщательном соблюдении методики. Оптимальный режим ПБД предполагает постепенное повышение давления от 20 до 26,7 кПа (от 150 до 200 мм рт. ст.), экспозицию от 30 до 60 с, которые соизмеряют со степенью расправления баллона и болевой реакцией пациента. ПБД помогает установить уровень и причину как стеноза при рецидивах АП после ЛКМТ, так и стеноза после пластики ПОД и фундопликации, и таким образом определить показания к повторной операции и ее объем.

Ключевые слова: ступенчатая пневматическая баллонная дилатация; ахалазия пищевода; стеноз пищеводно-желудочного перехода.

Abstract

Objective. To choose an optimal regime of pneumatic balloon dilatation (PBD) of esophago-gastric junction under roentgenological control in achalasia and stenosis after hiatoplasty and fundoplication.

Materials and methods. In 2007 - 2017 yrs PBD was accomplished in 34 patients with achalasia Stages I-II; 13 - with recurrence after laparoscopic cardiomyotomy (LCM); 14 - with functional dysphagia or achalasia after hiatoplasty and fundoplication. Results. Morbidity after PBD was absent. In all patients a remission was achieved through various terms and have depended upon dysphagia cause. The recurrences occurred were analyzed and compared with results of reoperations.

Conclusion. PBD is highly effective and is not accompanied by complications while follow-up of the procedure strictly. Optimal regime of PBD assumes a graded raising of pressure from 20 to $26.7 \mathrm{kPa}$ (from 150 to $200 \mathrm{~mm} \mathrm{Hg}$ ), exposition from 30 to $60 \mathrm{~s}$ which are compared with degree of the balloon blow grade and the patient's reaction on the pain occurred. PBD helps to establish the level and cause of stenosis in achalasia recurrence after LCM and after hiatal plasty and fundoplication, and, thus, - to determine indications for reoperation and its volume.

Keywords: graded pneumatic balloon dilatation; achalasia; stenosis of esophago-gastric junction.

Пневматическая баллонная дилатация (ПБд) показана при АП (у первичных пациентов с ранними стадиями заболевания, при рецидивах после кардиомиотомии), при СП после пластики ПОД и фундопликации. Реже ее используют при пептических стриктурах пищевода и стенозе пищеводно-кишечных анастомозов.

Существует несколько вариантов ПБД с использованием разных режимов. Наиболее безопасной является методика ступенчатой ПБД под рентгенологическим контролем.

Цель исследования: выбор оптимального режима ПБД пищеводно-желудочного перехода под рентгенологическим контролем при АП и СП после пластики ПОД и фундопликации

\section{Материалы и методы исследования}

На кафедре хирургии № 1 и кафедре роботизированной и эндоскопической хирургии Одесского национального медицинского университета с 2007 по 2017 г. ПБД выполнена 34 пациентам: 7 - с I, II стадией АП, 13 - с рецидивом после ЛКМТ и 14 - с длительной функциональной дисфагией или СП после пластики ПОД и фундопликации. Часть пациентов была направлена из других клиник. Из 34 пациентов 27 (79,4\%) выполнено два курса ПБД, 5 (14,7\%) - один, 2 (5,9\%) - три. В пределах одного курса 20 (58,8\%) пациентам выполнено по три процедуры, 14 (41,2\%) - по две. Всего выполнено 169 ПБД. Женщин было 26, мужчин - 8 в возрасте от 22 до 79 лет, средний возраст составил 42 года. 
Техника пневмокардиодилатации. На первом этапе проводили струну-проводник через пищеводно-желудочный переход с помощью эндоскопа. Сразу после этого больному выполняли ПБД под рентгенологическим контролем. Премедикация включала внутримышечные инъекции: 2 мл кеторолака, 1 мл димедрола, 5 мл ондансетрона.

ПБД выполняли под местной аэрозольной анестезией глотки 10\% раствором лидокаина. Больной находился в положении лежа на спине. Использовали баллоны для ахалазии фирмы Cook Medical (США) диаметром 35 мм и длиной 11 см с тремя рентгенконтрастными метками, располагающимися в верхней, средней и нижней его частях. Баллон через трехходовой кран присоединен к груше с манометром с одной стороны и со шприцом Жане для эвакуации воздуха из баллона с другой стороны. Перед введением баллона больной делал два глотка вазелинового масла. Баллон вводили по проводнику с несколькими глотками вазелинового масла в грудной отдел пищевода. Голову больного при этом поворачивали набок с целью эвакуации слюны как профилактики аспирации. Под контролем электронно-оптического преобразователя баллон во вздутом состоянии вводили в желудок, что определяли как по уровню тени диафрагмы, так и по ощущениям во время проведения баллона. Затем баллон подтягивали несколько назад таким образом, чтобы средняя рентгенконтрастная метка расположилась на уровне тени диафрагмы. После этого баллон раздували начиная с давления 20 кПа (150 мм рт. ст.). Обычно баллон принимал форму песочных часов, а его сужение соответствовало зоне максимального сужения пищеводно-желудочного перехода.

При СП после пластики ПОД у 12 (85,7\%) из 14 пациентов сужение баллона располагалось на уровне диафрагмы, у 2 (14,3\%) - ниже уровня диафрагмы; при рецидивах после ЛКМТ - на 2 - 3 см выше уровня диафрагмы у 10 (76,9\%) из 13 больных, у 3 (23,1\%) больных оно не определялось. Если сужение баллона располагалось между средней и крайней рентгеноконтрастными метками, он сдувался и позиционировался таким образом, чтобы его сужение располагалось на уровне средней рентгенконтрастной метки.

После позиционирования баллона выполняли собственно дилатацию. При первой дилатации в пределах курса создавали давление 20 - 22,7 кПа (150 - 170 мм рт. Ст.), при второй - 21,3 - 24,0 кПа (160 - 180 мм рт. ст.), при третьей - 24,0 - 26,7 кПа (180 - 200 мм рт. ст.). При этом стремились к полному расправлению баллона (ликвидации эффекта песочных часов), что обычно удавалось при третьей дилатации. Экспозиция составляла от 30 до 60 с, в среднем 45 с. Давление и экспозицию подбирали индивидуально в зависимости от болевой реакции пациента на процедуру. При появлении значительной боли нагнетание воздуха прекращали, экспозиция сокращалась. Если возникал позыв к рвоте или кашлю в процессе дилатации, баллон тотчас же сдували, чтобы избежать разрыва пищевода. Также зонд баллона в раздутом состоянии удерживали снаружи нежестко, позволяя больному двигаться в такт с дыхательными движениями диафрагмы. В процессе самой дилатации рентгенологический контроль практически не требовался, его использовали только при соскальзывании баллона в желудок для повторной коррекции. По окончанию процедуры баллон извлекали при повернутой набок голове пациента. Наличие следов крови на баллоне, а также умеренная болевая реакция пациента в процессе процедуры служили показателем адекватной дилатации.

После процедуры больного в лежачем положении транспортировали в палату, спустя 3 ч он начинал пить воду. Ненаркотические аналгетики вводили однократно не более чем 20\% пациентов. Интервал между дилатациями составлял 2 - 4 дня, в среднем 3 дня. Стационарное наблюдение требовалось в течение 1 сут после процедуры.

\section{Результаты}

В процессе проведения ПБД осложнений не было. Ни у одного из оперированных пациентов не было выраженной загрудинной боли и боли в горле. Перфорации, разрывов пищевода, кровотечения и аспирации не было. У 1 больного возникла нестабильная стенокардия, курс ПБД был прекращен.

У всех пациентов достигнута ремиссия на протяжении разных сроков, которые значительно различались между собой в зависимости от причин дисфагии. У пациентов с ранними стадиями АП средний период ремиссии составил 3 года (от 2 до 4 лет). Всем пациентам с рецидивами в дальнейшем выполнена ЛКМТ. Полная ремиссия достигнута у 11 (84,6\%) из 13 пациентов с рецидивом после ЛКМТ. Остальные 2 (14,4\%) пациента оперированы повторно. У этих больных сужение было на 2 - 3 см выше уровня диафрагмы вследствие недостаточной миотомии верхней части нижнего пищеводного сфинктера. Именно на этом уровне были рассечены гипертрофированные мышечные волокна сфинктера при повторной ЛКМТ, после чего дисфагия исчезла.

При СП после пластики ПОД и фундопликации у 9 (64,3\%) из 14 пациентов удалось добиться полной ликвидации стеноза. У остальных 5 (35,7\%) пациентов ремиссия сохранялась в среднем на протяжении 9 мес (от 3 мес до 1,5 года), после чего больные были оперированы повторно. У всех этих пациентов сужение пищевода локализовалось на уровне ПОД, что было подтверждено при повторных операциях.

\section{Обсуждение}

ПБД является эффективным методом лечения доброкачественных стенозов пищеводно-желудочного перехода. Ремиссия на протяжении $2-4$ лет сохраняется у 35 - 93\% пациентов, в среднем у 60\% [1 - 4]. Однако ПБД может сопровождаться грозными осложнениями: разрывом слизистой оболочки пищеводно-желудочного перехода с кровотечением, разрывом всей толщи стенки пищевода раздутым баллоном, перфорацией пищевода наконечником баллона с последующим развитием медиастинита или перитонита, а также аспирационной пневмонией. Частота осложнений составляет 0,5 - 5\%, в среднем 1,9\%, отдельные авторы сообщают о частоте $10 \%$ [2 - 5]. Стойкость и длительность ремиссии и частота осложнений напрямую зависят от методики ПБд, вида баллона, режима дилатации и прочих технических факторов $[1,6]$. 
Дилатация под эндоскопическим контролем, когда баллон располагается впереди эндоскопа, не позволяет точно позиционировать середину баллона на уровне стеноза, контролировать и соизмерять его расправление с создаваемым давлением. Поэтому при данной методике подбирают давление и время экспозиции, ориентируясь в основном на болевую реакцию пациента. Методика ПБД под рентгенологическим контролем позволяет не только четко позиционировать середину баллона на уровне стеноза и корректировать его положение в случае дислокации в процессе дилатации, но и, что является главным, соизмерять расправление баллона, создаваемое давление, экспозицию и болевую реакцию пациента одновременно. В современной литературе описана преимущественно ПБД под ренгтенологическим контролем [2, 4 - 6].

При использовании штатных баллонов, например баллонов для ахалазии фирмы Cook Medicall (США), сделанных из специального полимера и обеспечивающих строго циркулярную форму баллона, эффективность ПБД значительно выше, чем при использовании самодельных или неприспособленных для этого баллонов. Кроме того, специальная форма и материал наконечника штатного баллона позволяют легко проводить его через зону стеноза, избегая перфорации. Хотя основной мерой профилактики перфорации является выполнение дилатации по проводнику.

Существует также методика ПБД с параллельным расположением баллона и эндоскопа. Однако дилатацию под эндоскопическим контролем пациенты переносят хуже, чем ПБД под рентгенологическим контролем, и она наиболее опасна в плане разрыва пищевода и кровотечения, поскольку давление в зоне сужения распределяется неравномерно.

На основании нашего опыта можно судить о диагностических возможностях ПБД под рентгенологическим контролем. При рецидивах после ЛКМТ сужение баллона наблюдали на уровне $2-3$ см выше диафрагмы, что подтвердило недостаточность миотомии в верхней части нижнего пищеводного сфинктера и указало место его дополнительного рассечения при повторной операции. Сужение баллона на уровне ПОД после его пластики свидетельствовало о чрезмерной крурорафии или аллопластике в этом месте, на основании чего была определена цель повторной операции. Аналогичные данные приведены в литературе [1,7].

\section{Выводы}

1. Ступенчатая ПБД под рентгенологическим контролем высокоэффективна и не сопровождается осложнениями при тщательном соблюдении методики и технических нюансов.

2. Оптимальный режим дилатации предполагает постепенное повышение давления от 20 до 26,7 кПа (от 150 до 200 мм рт. ст.), экспозицию от 30 до 60 с, которые соизмеряются со степенью расправления баллона и болевой реакцией пациента.

3. ПБД помогает установить уровень и причину стеноза как при рецидивах АП после кардиомиотомии, так и при стенозе после пластики ПОД и фундопликации, позволяя таким образом определить показания к повторной операции и ее объем.

\section{References}

1. Fomin PD, Grubnik VV, Nikishaev VI, Malinovskij AV. Neopuholevye zabolevanija pishhevoda. Kiev: Biznes-Intellekt; 2008. 304 p. [In Russian].

2. Cheng JW, Li Y, Xing WQ, Lv HW, Wang HR. Laparoscopic Heller myotomy is not superior to pneumatic dilation in the management of primary achalasia: Conclusions of a systematic review and meta-analysis of randomized controlled trials. Medicine. 2017 Feb;96(7):e5525.

3. Moonen A, Annese V, Belmans A, Bredenoord AJ, Bruley des Varannes S, Costantini M. Long-term results of the European achalasia trial: a multicentre randomised controlled trial comparing pneumatic dilation versus laparoscopic Heller myotomy. Gut. 2016 May;65(5):732-9.

4. Uppal DS, Wang AY. Update on the endoscopic treatments for achalasia. World J Gastroenterol. 2016 Oct 21;22(39):8670-83.

5. Arora Z, Thota PN, Sanaka MR. Achalasia: current therapeutic options. Ther Adv Chronic Dis. 2017 Jun;8(6-7):101-8.

6. Illés A, Farkas N, Hegyi P, Garami A, Szabó I, Solymár M, et al. Is Heller myotomy better than balloon dilation? A meta-analysis. J Gastrointestin Liver Dis. 2017 Jun;26(2):121-7.

7. Granderath FA, Schweiger UM, Kamolz T, Pointner R. Dysphagia after laparoscopic antireflux surgery: a problem of hiatal closure more than a problem of the wrap. Surg Endosc. 2005 Nov;19(11):1439-46. 